\title{
Impacto de la optimización del tratamiento en pacientes con enfermedad por reflujo gastroesofágico que no responden a esomeprazol
}

\section{Impact of treatment optimization in patients with gastroesophageal reflux disease who do not respond to esomeprazole}

Janer Nelson Lozano-Martínez, William Alberto Otero-Regino, Hernando Marulanda-Ferníndez • Bogotá, D.C. (Colombia)

DOI: https://doi.org/10.36104/amc.2021.2041

\section{Resumen}

Introducción: la enfermedad por reflujo gastroesofágico (ERGE) afecta a una de cada ocho personas en Colombia. Sus síntomas característicos son pirosis y regurgitación. La piedra angular del tratamiento son los inhibidores de bomba de protones (IBP) con respuesta clínica en 58-80\%. En quienes no responden $75-90 \%$ tienen un trastorno funcional superpuesto y se podrían tratar adicionando neuromoduladores viscerales.

Objetivo: evaluar el impacto que tiene optimizar el tratamiento en pacientes con ERGE cuando no hay respuesta a esomeprazol (ESO).

Material y métodos: estudio prospectivo en pacientes sin respuesta clínica (más de dos episodios de reflujo por semana) tratados con ESO $40 \mathrm{mg}$ media hora antes del desayuno y simultáneamente recomendaciones para bajar de peso si IMC > 25, dejar de fumar y controlar el estrés, y finalmente aumentado la dosis de ESO a $40 \mathrm{mg}$ en ayunas y antes de la cena. Cuando se cumplió todo lo anterior y persistían los síntomas, se adicionó amitriptilina $12.5 \mathrm{mg}$ por la noche. Cada 12 semanas se evaluaba la respuesta.

Resultados: hubo 529 pacientes elegibles y 149 cumplieron los criterios de inclusión. Optimizando el tratamiento 111 pacientes tuvieron respuesta clínica sin la utilización de amitriptilina (74.5\%; IC95\% 67.2-81.4). En 22 se adicionó amitriptilina (14.8\%) y de estos respondieron 15 pacientes, $68.2 \%$ (IC95\% 47.04-89.32\%). En ocho pacientes hubo somnolencia (53.3\%). Se encontró relación entre el cumplimiento del tratamiento con IBP y la respuesta clínica $(\mathrm{p}<0.0001)$.

Conclusiones: en pacientes con ERGE la optimización del tratamiento con IBP mejora el 74.5\% (IC95\% 67.2-81.4) de los pacientes y la adición de amitriptilina a quienes no mejoran, logra mejorar el $68.2 \%$ de quienes no mejoraban con dos dosis de ESO.Con el manejo secuencial se logró mejoría acumulativa en el control de los síntomas de 85\% (IC95\% 78.6-90.4) de los pacientes. (Acta Med Colomb 2021; 46. DOI: https://doi.org/10.36104/amc.2021.2041).

Palabras clave: optimizar tratamiento, IBP, cumplimiento, respuesta clínica, neuromodulador.

\section{Abstract}

Introduction: gastroesophageal reflux disease (GERD) affects one out of eight people in Colombia. Its characteristic symptoms are heartburn and reflux. The cornerstone of treatment is proton pump inhibitors (PPIs), with a clinical response in 58-80\% of patients. Of those who do not respond, $75-90 \%$ have a superimposed functional disorder and could be treated by adding visceral neuromodulators.

Objective: to evaluate the impact of optimizing the treatment of patients with GERD when there is no response to esomeprazole (ESO).
Dr. Janer Nelson Lozano-Martínez: Internista. Residente de Gastroenterología, Universidad Nacional de Colombia; Dr. William Alberto Otero-Regino: Profesor Universidad Nacional de Colombia, Hospital Universitario Nacional. Gastroenterólogo Centro de Gastroenterología y Endoscopia Digestiva; Dr. Hernando Marulanda-Fernández: Profesor Universidad Nacional de Colombia, Hospital Universitario Nacional. Gastroenterólogo Centro de Gastroenterología y Endoscopia Digestiva. Bogotá, D.C. (Colombia).

Correspondencia: Dr. William Alberto OteroRegino. Bogotá, D.C. (Colombia). E-mail: waoteror@gmail.com

Recibido: 7/X/2020 Aceptado: 14/XII/2020 
Materials and methods: a prospective study in patients with no clinical response (more than two reflux episodes per week) who were treated with $40 \mathrm{mg}$ of ESO half an hour before breakfast along with the recommendation to lose weight if BMI >25, stop smoking and manage stress; and, finally, increasing the ESO dose to $40 \mathrm{mg}$ on an empty stomach and before dinner. When all of this was done and symptoms persisted, $12.5 \mathrm{mg}$ of amitriptyline were added at night. The response was evaluated every 12 weeks.

Results: a total of 529 patients were eligible and 149 met the inclusion criteria. With treatment optimization, 111 patients had a clinical response without using amitriptyline $(74.5 \%$; $95 \%$ CI $67.2-$ 81.4). Amitriptyline was added in 22 patients (14.8\%), 15 of whom responded $(68.2 \%$; $95 \% \mathrm{CI}$ 47.04-89.32\%). Eight patients experienced drowsiness (53.3\%). A relationship was found between PPI treatment compliance and clinical response $(\mathrm{p}<0.0001)$.

Conclusions: in patients with GERD, PPI treatment optimization improves 74.5\% (95\% CI 67.281.4) of the patients, and adding amitriptyline for those who do not improve achieves improvement in $68.2 \%$ of those who did not improve with two doses of ESO. Sequential management achieved a cumulative improvement in symptom control in 85\% (95\% CI 78.6-90.4) of the patients. (Acta

Med Colomb 2021; 46. DOI: https://doi.org/10.36104/amc.2021.2041).

Kew words: treatment optimization, PPI, compliance, clinical response, neuromodulator.

\section{Introducción}

La enfermedad por reflujo gastroesofágico (ERGE), se produce cuando el flujo retrógrado del contenido gástrico causa síntomas molestos y/o complicaciones esofágicas o extraesofágicas (1). Afecta 8-10\% de la población mundial y $11 \%$ de la población latinoamericana (2). En Colombia está presente en $12 \%$ (3). El diagnóstico se presume clínicamente cuando hay pirosis y/o regurgitación (4) y es conclusivo cuando al hacer endoscopia se encuentra esofagitis C o D (5) o cuando la endoscopia es negativa $(70 \%)$ y la impedanciometría con monitoreo de $\mathrm{pH}$ (impedaciometría-pH-metría) es positiva (5). Estos últimos pacientes constituyen lo que se denomina NERD (Non Erosive Reflux Disease) (5).

La piedra angular del tratamiento es inhibir la secreción de ácido con inhibidores de bomba de protones (IBP) y algunas medidas generales como disminuir el peso cuando el índice de masa corporal (IMC) es superior a $25 \mathrm{~kg} / \mathrm{m}^{2}$ $(6,7)$, dejar de fumar (8), y controlar el estrés (9). Otras modificaciones del estilo de vida, aunque frecuentemente recomendadas, carecen de evidencia de alta calidad que las respalde (10). Entre los diferentes IBP de primera generación, el esomeprazol (ESO) produce mayor inhibición de la secreción de ácido en el estómago y junto con el rabeprazol, tiene metabolismo independiente de actividad del CYP2C19 a nivel hepático $(11,12)$. En Colombia más del $75 \%$ de los individuos son metabolizadores rápidos o ultrarrápidos de los IBP (13). En pacientes con ERGE y erosiones esofágicas la respuesta se logra en $85-90 \%$ y cuando no hay esofagitis en $55-60 \%(14,15)$. En los pacientes tratados con IBP, sólo el 54-68\% cumplen el tratamiento $(16,17)$. El $84 \%$ de los pacientes que responden a IBP cumplen el tratamiento en contraste con $50 \%$ de los que no responden ( $\mathrm{p}<0.0001)$ (18). Cuando se cumple el tratamiento la eficacia es de $75 \%$ con una dosis al día y 80-90\% con dos dosis (19). El 75-90\% de los pacientes que no responden a IBP dos veces al día, tienen un trastorno funcional superpuesto como hipersensi- bilidad a reflujo o pirosis funcional (4, 20-22). No obstante, ese conocimiento, en ese grupo de pacientes los expertos y diferentes guías de manejo, recomiendan realizarles impedaciometría esofágica con monitoreo de $\mathrm{pH}$ esofágico (impedaciometría-pH-metría) (23-27) y más recientemente neuromoduladores viscerales a dosis bajas (28-30), especialmente antidepresivos tricíclicos (amitriptilina, imipramina) $(31,32)$, o inhibidores selectivos de la recaptación de serotonina (ISRS) como fluoxetina o citalopram $(30,33,34)$.

Teniendo en cuenta el alto costo de la impedanciometríapHmetría y que la mayoría de pacientes que no responden a dos dosis de IBP correctamente prescrito, tienen un trastorno funcional superpuesto, se decidió realizar el presente trabajo para determinar si la optimización del manejo de la ERGE, corrigiendo sucesivamente la prescripción incorrecta, la adopción de las medidas generales eficaces, dos dosis de IBP y finalmente la adición de neuromoduladores viscerales, podían controlar los síntomas de manera acumulativa.

\section{Material y métodos}

El presente es un estudio abierto prospectivo de intervención, basado en datos reales de la práctica clínica diaria, realizado en una cohorte de pacientes adultos mayores de 18 años con ERGE y atendidos en la consulta externa del Centro de Gastroenterología y Endoscopia Digestiva de Bogotá, adscrito al posgrado de gastroenterología de la Universidad Nacional de Colombia. Los criterios de inclusión fueron edad mayor o igual a 18 años, síntomas típicos de pirosis y regurgitación, tratamiento con ESO y falta de respuesta clínica al recibir tratamiento por 12 semanas. Los pacientes con ERGE y sintomáticos, a pesar del tratamiento, eran direccionados a una consulta especial atendida por uno de los autores (JL), quien a su vez los discutía con otro de los autores (WO). Los criterios de exclusión fueron embarazadas, falta de control de los síntomas típicos de reflujo, usar otro IBP diferente a ESO, intolerancia a ESO o amitriptili- 
na, enfermedad psiquiátrica en tratamiento (estar tomando Inhibidores selectivos de la recaptación de serotonina o antidepresivos tricíclicos), tener complicaciones de la ERGE (estenosis péptica, esófago de Barrett o cáncer esofágico, así mismo hallazgos endoscópicos que sugieran esofagitis eosinofílica (EE), cirugía previa del tracto digestivo alto, cáncer gastrointestinal actual o previo, trastornos motores del esófago; comorbilidad debilitante activa (enfermedad coronaria, arritmias cardiacas, EPOC, cirrosis, enfermedad renal crónica, esclerosis, gastroparesia).

\section{Intervenciones}

En la visita inicial, se identificaban las características de los pacientes y según cada caso, se corregía la prescripción del ESO y se realizaban recomendaciones generales: bajar de peso, dejar de fumar y reducir el estrés. Al lograr el cumplimiento y no obtener respuesta clínica se les duplicaba la dosis de esomeprazol y se evaluaban en las siguientes 12 semanas. Si en la evaluación de control no había respuesta al tratamiento se realizaba nuevamente la optimización y se programaba una nueva evaluación. Se consideró respuesta al tratamiento la desaparición de los síntomas o disminución de la frecuencia de los mismos a menos de dos veces por semana. La respuesta clínica se determinó mediante llamada telefónica, consulta y revisión de la historia clínica.

\section{Análisis estadístico}

Las variables cuantitativas se presentan como medida de resumen y dispersión, las variables cualitativas se presentan en números absolutos y proporciones. La prueba de Mc Nemar se empleó para evaluar la respuesta clínica y el cumplimiento, la prueba $t$ de student para comparar variables cuantitativas pareadas y la prueba de $X^{2}$ para comparaciones entre variables cualitativas, se tuvo en cuenta un valor de p $<0.05$ como estadísticamente significativo. Se realizó un análisis de sensibilidad de las pérdidas y se consideró a estas como no cumplimiento o como no respuesta clínica al tratamiento. El análisis estadístico se realizó con el programa SPSS-26.

\section{Resultados}

Se identificaron 529 pacientes elegibles, se excluyeron 380 y finalmente se seleccionaron 149 (Figura 1).

\section{Características generales de los pacientes}

El promedio de edad de los pacientes del estudio fue de 59.6 años \pm 12.5 ; La mayoría estaban en el grupo de edad entre 60-74 años (43.2\%) seguidos por el grupo de 35-59 años $(42.3 \%)$, las mujeres correspondieron al $83.2 \%$. La característica más común fue el sobrepeso y la obesidad (38.9 y $24.8 \%$ respectivamente). Los hallazgos endoscópicos más frecuentemente encontrados fueron la esofagitis grado A (47.0\%); la hernia hiatal se presentó en $14.1 \%$ de los participantes. En la Tabla 1 se resumen las características de los pacientes del estudio.

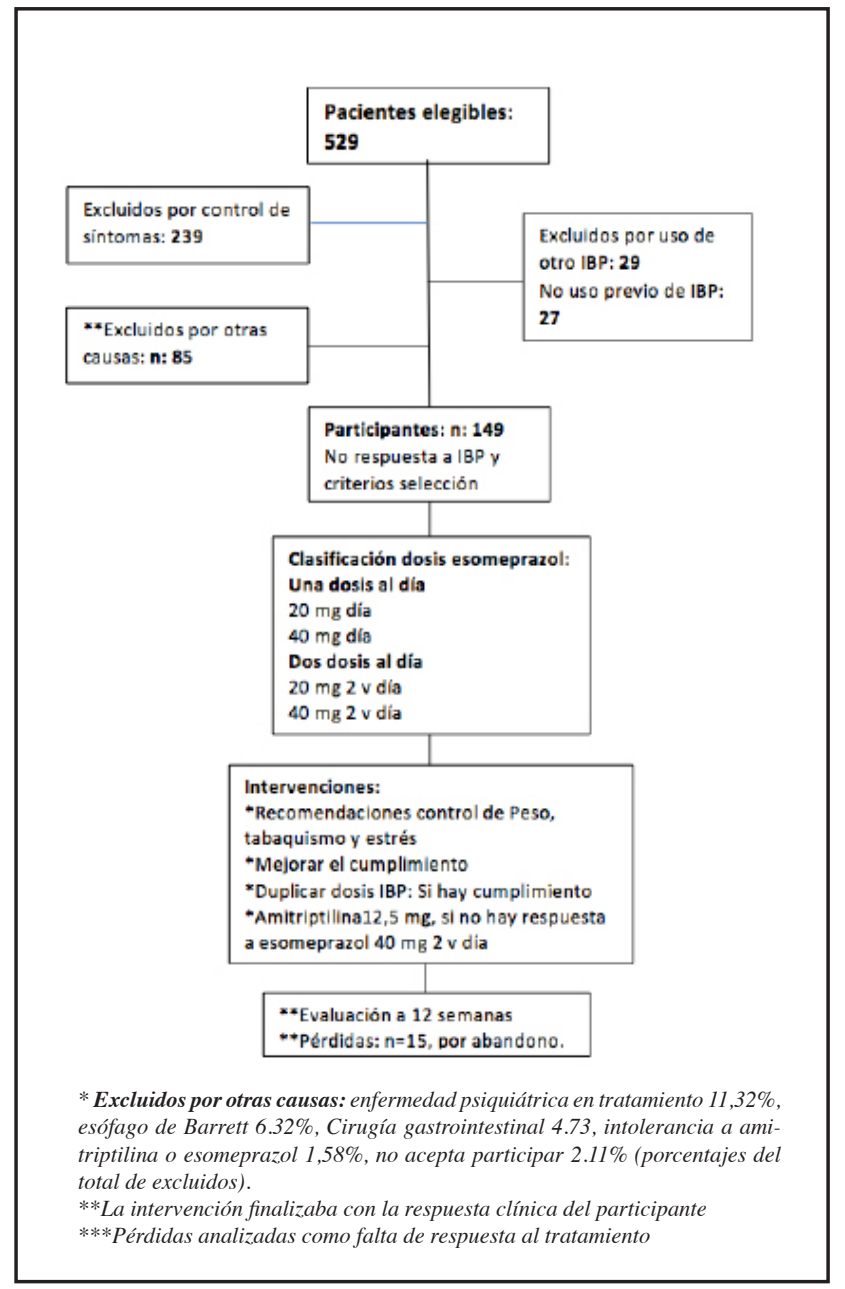

Figura 1: Pacientes incluidos y excluidos en el estudio.

El $63.1 \%$ de los participantes no tomaban de forma adecuada el tratamiento; la dosis de esomeprazol que más se asoció a una baja adherencia fue la de $40 \mathrm{mg}$ dos veces al día $(76.6 \%)$ (Tabla 2).

\section{Pérdidas del estudio}

El total de pérdidas fue de 15 participantes (10.1\%) un porcentaje menor al esperado (20\%). Las pérdidas se produjeron por abandono del participante y fueron analizadas como no respuesta o no cumplimiento del tratamiento.

\section{Respuesta general}

La evaluación de la intervención de optimización se realizó desde agosto de 2019 hasta marzo del 2020. No se encontró relación entre la edad y el género con la respuesta clínica al tratamiento, ni con el cumplimiento. Dentro de las recomendaciones en la optimización del tratamiento para reflujo refractario, la reducción del peso en obesos es un objetivo clave (Tabla 3).

Los pacientes con sobrepeso lograron una reducción en promedio de $0.5 \mathrm{~kg}$ (IC95\% -0.1-1.1) y una variación del IMC de 0.2 (IC95\% -0.04-0.45). Para los obesos la media de 
Tabla 1. Características generales de los pacientes.

\begin{tabular}{|c|c|c|}
\hline \multirow{2}{*}{ Característica } & \multicolumn{2}{|c|}{ Incluidos en estudio ( $n=149)$} \\
\hline & Media & Desviación estándar \\
\hline Edad & 59,6 & \pm 12.5 \\
\hline Peso & 67,3 & \pm 11.9 \\
\hline Talla & 1,6 & \pm 0.07 \\
\hline IMC & 27,0 & \pm 4.5 \\
\hline Género & Número & Porcentaje \\
\hline Femenino & 124 & $83.2 \%$ \\
\hline Ocupación & Número & Porcentaje \\
\hline Pensionado & 62 & $41.6 \%$ \\
\hline Docente & 59 & $39.6 \%$ \\
\hline Hogar & 22 & $14.8 \%$ \\
\hline Otros & 6 & $4.0 \%$ \\
\hline IMC & Número & Porcentaje \\
\hline IMC normal & 54 & $36.2 \%$ \\
\hline Sobrepeso & 58 & $38.9 \%$ \\
\hline Obeso & 37 & $24.8 \%$ \\
\hline Estilo de vida & Número & Porcentaje \\
\hline Fumadores & 3 & $2.01 \%$ \\
\hline Estrés & 87 & $58.4 \%$ \\
\hline Dosis esomeprazol & Número & Porcentaje \\
\hline $20 \mathrm{mg} /$ día & 20 & $13.4 \%$ \\
\hline $20 \mathrm{mg} / 2$ v día & 24 & $16.1 \%$ \\
\hline $40 \mathrm{mg} /$ día & 41 & $27.5 \%$ \\
\hline $40 \mathrm{mg} / 2$ v día & 64 & $43.0 \%$ \\
\hline Esofagitis & Número & Porcentaje \\
\hline No esofagitis & 63 & $42.3 \%$ \\
\hline Grado A & 70 & $47.0 \%$ \\
\hline Grado B & 10 & $6.7 \%$ \\
\hline Grado C & 5 & $3.4 \%$ \\
\hline Grado D & 1 & $0.7 \%$ \\
\hline Hernia hiatal & 21 & $14.1 \%$ \\
\hline
\end{tabular}

reducción del peso corporal fue $1.43 \mathrm{Kg}$ (IC95\% 0.36-2.51) y del IMC de 0.6 (IC95\% 0.17-1.04).

\section{Respuesta clínica al esomeprazol}

La respuesta clínica al esomeprazol de todos los pacientes, incluso aquellos que no cumplieron los criterios para el uso de amitriptilina, está detallada según la dosis empleada en la Tabla 4. Es claro que al optimizar la terapia, la respuesta clínica mejoró de forma significativa. El grupo de $20 \mathrm{mg}$ dos veces al día tuvo el más alto porcentaje de respuesta clínica (93.33\%) los cambios en los otros grupos se resumen en la Tabla 4.

\section{Respuesta clínica a amitriptilina y dosis máxima de esomeprazol}

Veintisiete pacientes no tuvieron respuesta al tratamiento optimizado con esomeprazol a dosis máxima. De este grupo, 5 no continuaron con el protocolo, los 22 restantes recibieron tratamiento con amitriptilina $12.5 \mathrm{mg}$ noche, más esomeprazol $40 \mathrm{mg}$ cada 12 horas; de éstos, 15 tuvieron respuesta clínica $(68.18 \%$; $\mathrm{p}<0.001)$; el evento adverso más frecuente fue somnolencia diurna (ocho pacientes 53.3\%; $\mathrm{p}=0.004)$ (Tabla 5).

Con el manejo secuencial desde la optimización del IBP hasta la adición de amitriptilina, se logró mejoría acumulativa en el control de los síntomas de 85\% (IC95\% 78.6-90.4) de los pacientes. Al final de la intervención se observó un incremento en el cumplimiento del tratamiento de $44.3 \%(p<0.001)$. Se encontró una relación directa entre la adherencia y la respuesta al tratamiento (con o sin amitriptilina; $\mathrm{p}<0.001$ respectivamente).

\section{Discusión}

El presente trabajo se ha realizado en una cohorte de pacientes de consulta externa rutinaria, en el cual no se tienen suficientes recursos económicos para estudiar exhaustivamente los pacientes con ERGE. Adicionalmente hay evidencia de que la falta de respuesta al tratamiento incluye en muchos pacientes una inadecuada prescripción de los IBPs $(35,36)$. Otras causas incluyen falta de adherencia al tratamiento (16-18), insuficiente inhibición de ácido con una sola dosis al día (19), biodisponibilidad de los IBP $(11,12)$, reflujo gastroesofágico no ácido (37), esofagitis

Tabla 2. Dosis de Esomeprazol y cumplimiento del tratamiento al ingresar en el estudio.

\begin{tabular}{|c|c|c|c|c|c|c|}
\hline Dosis Esomeprazol & Cumplimiento & $\%$ & No cumplimiento & $\%$ & Total & $\%$ \\
\hline 20 mg/día & 10 & 50.0 & 10 & 50.0 & 20 & 13.4 \\
\hline 40 mg/día & 19 & 46.3 & 22 & 53.7 & 41 & 27.5 \\
\hline $20 \mathrm{mg} / 2$ v día & 11 & 45.8 & 13 & 54.2 & 24 & 16.1 \\
\hline $40 \mathrm{mg} / 2$ v día & 15 & 23.4 & 49 & 76.6 & 64 & 43.0 \\
\hline Total & 55 & 36.9 & 94 & 63.1 & 149 & 100.0 \\
\hline
\end{tabular}


Tabla 3. Cambios en el peso e IMC

\begin{tabular}{|l|c|c|c|c|}
\hline $\begin{array}{l}\text { Categoría } \\
\text { IMC }\end{array}$ & Item & Media Inicio & Media Final & p \\
\hline \multirow{2}{*}{ Normal } & Peso & $58.50 \pm 7.15$ & $58.81 \pm 7.45$ & 0.229 \\
\cline { 2 - 5 } & IMC & $22.74 \pm 1.78$ & $22.87 \pm 1.94$ & 0.254 \\
\hline \multirow{2}{*}{ Sobrepeso } & Peso & $66.05 \pm 6.07$ & $65.55 \pm 6.58$ & 0.100 \\
\cline { 2 - 5 } & IMC & $27.17 \pm 1.42$ & $26.97 \pm 1.63$ & 0.113 \\
\hline \multirow{2}{*}{\begin{tabular}{l} 
Obesidad \\
\cline { 2 - 5 }
\end{tabular}} & Peso & $82.3 \pm 10.1$ & $80.8 \pm 10.8$ & 0.007 \\
\cline { 2 - 5 } & $\mathrm{IMC}$ & $33.1 \pm 3.0$ & $32.5 \pm 3.1$ & 0.007 \\
\hline \multicolumn{7}{|l}{$\begin{array}{l}\text { Peso en Kg; IMC Kg/m } 2: \text { normal: }<25 \mathrm{~kg} / \mathrm{m}^{2}, \text { sobrepeso: } 25-29.9 \mathrm{~kg} / \mathrm{m}^{2}, \text { obesidad } \\
\geq 30 \mathrm{Kg} / \mathrm{m}^{2} .\end{array}$}
\end{tabular}

Tabla 4. Respuesta clínica esomeprazol según dosis específica.

\begin{tabular}{|l|c|c|c|c|c|}
\hline Dosis esomeprazol & Respuesta & $\%$ & Sin Respuesta & $\%$ & Total \\
\hline $20 \mathrm{mg}$ día* & 6 & 54.55 & 5 & 45.45 & 11 \\
\hline $40 \mathrm{mg}$ día* & 19 & 79.17 & 5 & 20.83 & 24 \\
\hline $20 \mathrm{mg} 2$ veces día* & 14 & 93.33 & 1 & 6.67 & 15 \\
\hline $40 \mathrm{mg} 2$ veces día* & 72 & 72.73 & 27 & 27.27 & 99 \\
\hline Total & 111 & 74.50 & 38 & 25.50 & 149 \\
\hline
\end{tabular}

Tabla 5. Respuesta clínica amitriptilina y dosis máximas de esomeprazol.

\begin{tabular}{|l|c|c|c|c|c|}
\hline \multicolumn{1}{|c|}{ Seguimiento } & Respuesta & $\%$ & $\begin{array}{c}\text { Sin } \\
\text { Respuesta }\end{array}$ & $\%$ & Total \\
\hline Primer control & 13 & 86.67 & 2 & 9.09 & 15 \\
\hline Segundo control & 1 & 16.67 & 5 & 22.73 & 6 \\
\hline Tercer control & 1 & 100.00 & 0 & 0.00 & 1 \\
\hline Total & 15 & 68.18 & 7 & 31.82 & 22 \\
\hline
\end{tabular}

eosinofílica (37), acalasia (37), obesidad (7), tabaquismo (8) y estrés (9), o también un trastorno funcional sobrepuesto o uno de estos trastornos como entidad primaria $(20,21)$. Por lo anterior, la evaluación de los pacientes refractarios debe comenzar con una adecuada valoración de los síntomas, esófago sin alteraciones endoscópicas o con esofagitis erosiva (C o D de los Ángeles) (5). Además, determinar la presencia o no de obesidad, estrés, tabaquismo, verificación del cumplimiento de la formulación de los IBPs etc. (7-9, 16-19).

En este estudio se diseñó un protocolo de intervenciones para los pacientes clasificados con reflujo refractario por inadecuada respuesta al tratamiento con esomeprazol, el primer paso fue la optimización del tratamiento, logrando una mejoría sintomática en $75 \%$ de los pacientes previamente clasificados como refractarios, con resultados similares a los reportados por Fass y Shapiro (15). La optimización tiene dos pilares fundamentales: el primero es garantizar la adherencia, con lo que se obtiene una respuesta clínica en $71.4 \%$ de los casos. No obstante, hay un porcentaje de pacientes que no mejoran y el siguiente paso es incrementar la dosis del IBP (dos veces al día), logrando mejoría en $74.5 \%$ de los pacientes, resultados más favorables que los reportados en el estudio de Fass y Murthy (38), que al incrementar la dosis a dos veces al día lograron resolución de los síntomas solo en $20 \%$ (39).

En el grupo de pacientes que no responden a pesar de las estrategias de optimización, adicionamos un neuromodulador visceral (amitriptilina), teniendo en cuenta que estudios previos han demostrado que cuando un paciente recibe dos dosis correctas de IBP, se logra una profunda supresión de ácido y en $90 \%$ de ellos la persistencia de los síntomas se debe a un trastorno funcional sobrepuesto (4). Charbel y col. (40), encontraron que cuando los pacientes reciben dos dosis de omeprazol, sólo $3.8 \%$ tenían pH-metría esofágica anormal. En otro estudio se encontró que la probabilidad de una pH-metría normal, utilizando dosis dobles de IBP es 11 (IC95\% 4.3-30.1 p<0.01) (40). En nuestro estudio el 68.2\% de los pacientes ( 15 de 22 ), que tenían dos dosis de esomeprazol tuvieron mejoría clínica al adicionarles amitriptilina. Resultados similares encontraron Faruqui y col (32) quienes, al adicionar amitriptilina a pantoprazol, mejoró la pirosis en $65 \%$ de los pacientes y la regurgitación en $94.2 \%$. En ese estudio no se reportaron efectos adversos, en contraste con el presente estudio, en el cual 53\% de los pacientes manifestó somnolencia leve, sin que le interfiriera con sus actividades cotidianas. Recientemente Abdallah y cols (20) encontraron que al comparar los pacientes con y sin respuesta a una dosis de IBP, la impedancio-pH-metría fue similar en ambos grupos con respecto al tipo de reflujo (ácido, débilmente ácido y débilmente alcalino), pero el 75\% de los pacientes sin respuesta al IBP tenían un trastorno esofágico funcional sobrepuesto. Estos resultados justificarían adicionar un neuromodulador cuando los síntomas persisten a pesar del IBP. En nuestro trabajo, empíricamente se adicionó una segunda dosis de IBP basados en hallazgos previos de que al dar dos dosis de IBP más de $96 \%$ de los pacientes logran suprimir la secreción de ácido (40) y si no había respuesta, se adicionó amitriptilina teniendo en cuenta la posibilidad de coexistencia de un trastorno funcional (4). El abordaje de pacientes con pirosis refractaria es complejo y se han ensayado diversos enfoques y estrategias. Este año, Vaezi y cols (42), reportaron que la utilización de un quelante de sales biliares IW3718 (Una presentación especial de colesevelam que le permite mantenerse en el estómago y atrapar los ácidos biliares) a dosis de 1.5 gramos dos veces al día, a la semana ocho, logró disminución del puntaje de pirosis de $11.9 \%$ comparado con placebo ( 58 vs $46 \%$ ) ( $\mathrm{p}=0.02)$. En ese estudio, los pacientes incluidos debían tener síntomas de ERGE refractario a una dosis de IBP en las últimas ocho semanas (42). Esos resultados podrían explicarse por el efecto nocivo que tienen los ácidos biliares conjugados en 
el epitelio esofágico (43-44); sin embargo, la ganancia sobre placebo es solo $12 \%$. Consideramos que se necesitarían más estudios para determinar si esta forma novedosa de colesevelam sería más eficaz que adicionar una segunda de IBP para una satisfactoria supresión del ácido (19-22). Otra estrategia empírica para el tratamiento de pirosis refractaria es el tratamiento quirúrgico (45). Recientemente se comparó funduplicatura de Nissen por laparoscopia versus omeprazol $20 \mathrm{mg}$ en ayunas y antes de cena más baclofén (inhibidor de la relajación del esfínter esofágico inferior), más imipramina en pacientes con pirosis refractaria (45). El tratamiento quirúrgico fue más eficaz que el tratamiento médico (67 vs $28 \%, \mathrm{p}=0.007$ ); sin embargo, en cada grupo $40-50 \%$ de los pacientes tenían hipersensibilidad visceral y el resto, reflujo anormal de ácido (impedancio-pH-metría). Este último hallazgo, demuestra que no había un adecuado control de la secreción de ácido y por lo tanto, estaría indicado duplicar la dosis de IBP (19). Si la secreción de ácido no estaba correctamente suprimida, sería preferible aumentar la dosis de IBP en vez de realizar una cirugía. La pirosis refractaria a IBP, no es indicación de cirugía (12). El hecho de que casi la mitad de los pacientes tuvieran hipersensibilidad esofágica, no se descarta que la superioridad de la cirugía se deba a un efecto placebo. Por lo anterior, consideramos que los hallazgos de esa investigación no pueden ser extrapolables a otras poblaciones. Adicionalmente se ignora la prevalencia de metabolizadores rápidos o ultrarrápidos de IBP en esa población. Con esta información, estaría indicado utilizar IBPs no influidos por el CYP $(11,12)$ si la prevalencia de esos genotipos es alta. Varios autores han destacado, que el tratamiento de la ERGE no es solamente suprimir la secreción de ácido con dosis mayores de $\operatorname{IBP}(4,32)$. El concepto fisiopatológico básico clásico de la ERGE, que la considera secundaria a una alteración en la relajación del esfínter esofágico, permitiendo el ascenso de ácido clorhídrico y bilis al esófago (46); sin embargo, la ERGE es mucho más compleja y hay múltiples fenotipos individuales (46).

Consideramos que nuestro trabajo, muestra una alternativa empírica para el manejo cotidiano de los pacientes con ERGE y estos resultados, adicionan nueva evidencia para no apresurarse a utilizar de manera innecesaria impedanciopH-metría en los pacientes que no responden inicialmente a IBP, como ha sido sugerido antes $(23,26,27,41)$.

Nuestro trabajo tiene limitaciones. No se tomaron biopsias esofágicas a los pacientes que seguían con síntomas a pesar del neuromodualor, para descartar esofagitis eosinofílica, muestra pequeña, no haber iniciado con pacientes de primera vez y haberlos seguido desde el inicio del IBP con dosis única y en adelante determinar en cada modificación del tratamiento cuánto era el rendimiento adicional de cada intervención sucesiva para poder establecer el rendimiento con este abordaje en la consulta externa, de la vida real de un país subdesarrollado. No obstante, hemos encontrado que en pacientes que no responden al tratamiento con IBP, optimizando el tratamiento se logra mejoría en $85 \%$ de los pacientes y por lo tanto consideramos que antes de solicitar impedaciometría-pH-metría esofágica, se debería optimizar el tratamiento con la estrategia propuesta en este trabajo.

\section{Conclusión}

El presente enfoque terapéutico de la ERGE representa el primer estudio en nuestro país y en Latinoamérica, que demuestra el beneficio de la optimización del IBP evitando estudios esofágicos costosos. El éxito acumulado del $85 \%$ es muy importante en manejo cotidiano de estos pacientes. Se requieren más investigaciones similares y con mayor número de pacientes, para determinar si este enfoque terapéutico sería una alternativa para un país con limitaciones económicas en salud.

\section{Referencias}

1. Vakil N, Van Zanten S V., Kahrilas P, Dent J, Jones R, Bianchi LK, et al. The Montreal definition and classification of gastroesophageal reflux disease: A global evidence-based consensus. Am J Gastroenterol. 2006;101(8):1900-20.

2. GBD 2017 Gastro-oesophageal Reflux Disease Collaborators. The global, regional, and national burden of gastro-oesophageal reflux disease in 195 countries and territories, 1990-2017: a systematic analysis for the Global Burden of Disease Study 2017. Lancet Gastroenterol Hepatol. 2020;5(6):561-581.

3. Páramo-hernández DB, Albis R, Galiano MT, Molano B De, Rincón R, Pineda-ovalle LF, et al. Prevalence of Gastro-Esophageal Reflux Symptoms and Associated Factors: A Population Survey in the Principal Citie. Rev Colomb Gastroenterol. 2016;31(4):5-12.

4. Gyawali CP, Fass R. Management of Gastroesophageal Reflux Disease. Gastroenterology. 2018;154(2):302-18.

5. Prakash Gyawali C, Kahrilas PJ, Savarino E, Zerbib F, Mion F, Smout AJPM, et al. Modern diagnosis of GERD: The Lyon Consensus. Gut. 2018;67(7):1351-62.

6. Hallan A, Bomme M, Hveem K, Møller-Hansen J, Ness-Jensen E. Risk factors on the development of new-onset gastroesophageal reflux symptoms. A population-based prospective cohort study: The HUNT study. Am J Gastroenterol. 2015;110(3):393-400.

7. Ness-Jensen E, Lindam A, Lagergren J, Hveem K. Weight loss and reduction in gastroesophageal reflux. a prospective population-based cohort study: The HUNT study. Am J Gastroenterol. 2013;108(3):376-82.

8. Ness-Jensen E, Lindam A, Lagergren J, Hveem K. Tobacco smoking cessation and improved gastroesophageal reflux: A prospective population-based cohort study: The HUNT study. Am J Gastroenterol. 2014;109(2):171-7.

9. Song EM, Jung HK, Jung JM. The association between reflux esophagitis and psychosocial stress. Dig Dis Sci. 2013;58(2):471-7.

10. Castillo R, Otero W TA. Impacto de las medidas generales en el tratamiento del reflujo gastroesofágico: una revisión basada en la evidencia. Rev Col Gastroenterol. 2015;30(4):431-6.

11. Schwab M, Klotz U, Hofmann U, Schaeffeler E, Leodolter A, Malfertheiner P, et al. Esomeprazole-induced healing of gastroesophageal reflux disease is unrelated to the genotype of CYP2C19: Evidence from clinical and pharmacokinetic data. Clin Pharmacol Ther. 2005;78(6):627-34.

12. Hillman L, Yadlapati R, Thuluvath AJ, Berendsen MA, Pandolfino JE. A review of medical therapy for proton pump inhibitor nonresponsive gastroesophageal reflux disease. Dis Esophagus. 2017;30(9):1-15.

13. Arévalo Galvis A, Trespalacios Rangel AA, Otero Regino W. Personalized therapy for Helicobacter pylori: CYP2C19 genotype effect on first-line triple therapy. Helicobacter. 2019;24(3):e12574.

14. Chiba N, De Gara CJ, Wilkinson JM, Hunt RH. Speed of healing and symptom relief in grade II to IV gastroesophageal reflux disease: A meta-analysis. Gastroenterology. 1997;112(6):1798-810.

15. Fass R, Shapiro M, Dekel R, Sewell J. Systematic review: proton-pump inhibitor failure in gastro-oesophageal reflux disease-where next? Aliment Pharmacol Ther. 2005 Jul;22(2):79-94.

16. Van Soest EM, Siersema PD, Dieleman JP, Sturkenboom MCJM, Kuipers EJ.Persistence and adherence to proton pump inhibitors in daily clinical practice. Aliment Pharmacol Ther. 2006;24(2):377-85.

17. Gosselin A, Luo R, Lohoues H, Toy E, Lewis B, Crawley J, et al. The impact of proton pump inhibitor compliance on health-care resource utilization and costs in patients with gastroesophageal reflux disease. Value Heal. 2009;12(1):34-9. 
18. Dickman R, Boaz M, Aizic S, Beniashvili Z, Fass R, Niv Y. Comparison of clinical characteristics of patients with gastroesophageal reflux disease who failed proton pump inhibitor therapy versus those who fully responded. J Neurogastroenterol Motil. 2011;17(4):387-94.

19. Richter JE. How to manage refractory GERD. Nat Clin Pract Gastroenterol Hepatol. 2007;4(12):658-64.

20. Abdallah J, George N, Yamasaki T, Ganocy S, Fass R. Most Patients With Gastroesophageal Reflux Disease Who Failed Proton Pump Inhibitor Therapy Also Have Functional Esophageal Disorders. Clin Gastroenterol Hepatol. 2019;17(6):1073-1080.e1.

21. Roman S, Keefer L, Imam H, Korrapati P, Mogni B, Eident K, et al. Majority of symptoms in esophageal reflux PPI non-responders are not related to reflux. Neurogastroenterol Motil. 2015 Nov 1;27(11):1667-74.

22. Fass OZ, Fass R. Overlap Between GERD and Functional Esophageal Disordersa Pivotal Mechanism for Treatment Failure. Curr Treat Options Gastroenterol. 2019;17(1):161-164.

23. Katz PO, Gerson LB, Vela MF. Guidelines for the diagnosis and management of gastroesophageal reflux disease. Am J Gastroenterol. 2013;108(3):308-329.

24. Iwakiri K, Kinoshita Y, Habu Y, et al. Evidence-based clinical practice guidelines for gastroesophageal reflux disease 2015. J Gastroenterol. 2016;51(8):751-767.

25. Hunt R, Armstrong D, Katelaris P, et al. World Gastroenterology Organisation Global Guidelines: GERD Global Perspective on Gastroesophageal Reflux Disease. J Clin Gastroenterol. 2017;51(6):467-478.

26. Trudgill NJ, Sifrim D, Sweis R, et al. British Society of Gastroenterology guidelines for oesophageal manometry and oesophageal reflux monitoring. Gut. 2019;68(10):1731-1750.

27. Savarino E, Bredenoord AJ, Fox M, et al. Expert consensus document: Advances in the physiological assessment and diagnosis of GERD. Nat Rev Gastroenterol Hepatol. 2017;14(11):665-676).

28. Aziz Q, Fass R, Gyawali CP, Miwa H, Pandolfino JE, Zerbib F. Esophageal disorders. Gastroenterology. 2016;150(6):1368-79.

29. Hungin APS, Molloy-Bland M, Scarpignato C. Revisiting Montreal: New Insights into Symptoms and Their Causes, and Implications for the Future of GERD. Am J Gastroenterol. 2019;114(3):414-21.

30. Ostovaneh MR, Saeidi B, Hajifathalian K, Farrokhi-Khajeh-Pasha Y, Fotouhi A, Mirbagheri SS, et al. Comparing omeprazole with fluoxetine for treatment of patients with heartburn and normal endoscopy who failed once daily proton pump inhibitors: Double-blind placebo-controlled trial. Neurogastroenterol Motil. 2014;26(5):670-8

31. Weijenborg PW, de Schepper HS, Smout AJPM, Bredenoord AJ. Effects of Antidepressants in Patients With Functional Esophageal Disorders or Gastroesophageal Reflux Disease: ASystematic Review. Clin Gastroenterol Hepatol. 2015;13(2):251-259.e1.
32. Faruqui AA. Gastroesophageal Reflux Disease Associated With Anxiety: Efficacy and Safety of Fixed Dose Combination of Amitriptyline and Pantoprazole. Gastroenterol Res. 2017;10(5):301-4.

33. Viazis N, Keyoglou A, Kanellopoulos AK, Karamanolis G, Vlachogiannakos J, Triantafyllou K, et al. Selective serotonin reuptake inhibitors for the treatment of hypersensitive esophagus: A randomized, double-blind, placebo-controlled study. Am J Gastroenterol. 2012;107(11):1662-7.

34. Broekaert D, Fischler B, Sifrim D, Janssens J, Tack J. Influence of citalopram, a selective serotonin reuptake inhibitor, on oesophageal hypersensitivity: A doubleblind, placebo-controlled study. Aliment Pharmacol Ther. 2006;23(3):365-70.

35. Gunaratnam NT, Jesup TP, Inadomi J, et al. Suboptimal proton pump inhibitor dosin is prevalent in patiens whih poorly controlled gastro-oesophageal reflux disease. Aliment Pharmacol Ther 2006; 23: 1473-7.

36. Chey WD, Inadomi JM, Booher AM, Sharma VK, Fendrick AM, Howden CW. Primary-care physicians' perceptions and practices on the management of GERD: results of a national survey. Am J Gastroenterol. 2005;100(6):1237-1242).

37. Rimon Sobhi AZZAM. Are the persistent symptoms to proton pump inhibitor therapy due to refractory gastroesophageal reflux disease or to other disorders? Arq Gastroenterol, 2018: 55 (Supl): 85-91

38. Fass R, Murthy U, Hayden CW, et al. Omeprazole $40 \mathrm{mg}$ once a day is equally effective as lansoprazole $30 \mathrm{mg}$ twice a day in symptom control of patients with gastro-oesophageal reflux disease (GERD) who are resistant to conventional-dose lansoprazole therapy-a prospective, randomized, multi-centre study. Aliment Pharmacol Ther. 2000;14(12):1595-1603.

39. Ates F, Vaezi MF. New Approaches to Management of PPI-Refractory Gastroesophageal Reflux Disease. Curr Treat Options Gastroenterol. 2014;12(1):18-33.

40. Charbel s, Khandwala F, Vaezi MF. The role of esophageal $\mathrm{pH}$ monitoring in symptomatic patients on PPI therapy. Am J Gastroenterol.2005;100(2):283-289.

41. Yadlapati R, DeLay K. Proton Pump Inhibitor-Refractory Gastroesophageal Reflux Disease. Med Clin North Am. 2019;103(1):15-27.

42. Vaezi MF, Fass R, Vakil N, et al. IW-3718 Reduces Heartburn Severity in Patients With Refractory Gastroesophageal Reflux Disease in a Randomized Trial. Gastroenterology 2020;158:2093-2103.

43. Nehra D, Howell P, Williams CP, et al. Toxic bile acids in gastro-oesophageal reflux disease: influence of gastric acidity. Gut 1999;44:598-602.

44. Gadacz T, Zuidema G. Bile acid composition in patients with and without symptoms of postoperative reflux gastritis. Am J Surg 1978;135:48-52.

45. Spechler SJ, Hunter JG, Jones KM, Lee R, Smith BR, Mashimo H, et al. Randomized Trial of Medical versus Surgical Treatment for Refractory Heartburn. N Engl J Med. 2019 Oct 17;381(16):1513-1523.

46. Katzka DA,John E. Pandolfino JE, Kahrilas PJ. Phenotypes of Gastroesophageal Reflux Disease: Where Rome, Lyon, and Montreal Meet. Clinical Gastroenterology and Hepatology 2020;18:767-776. 